\title{
CARACTERIZACIÓN DE LAS PERSPECTIVAS DOCENTES DEL PROFESORADO DE SECUNDARIA A PARTIR DEL ANÁLISIS DE LAS VARIABLES EDUCATIVAS RELACIONADAS CON LA ACCIÓN Y EL PENSAMIENTO DOCENTE ${ }^{1}$
}

\author{
Joan A. Traver, Auxiliadora Sales, Fernando Doménech y Odet Moliner
}

Universitat Jaume I, España

\section{FUNDAMENTACIÓN TEÓRICA}

\subsection{Estilos de enseñanza y pensamiento del profesorado: las perspectivas docentes}

Podemos definir los estilos de enseñanza como las diferentes formas que tienen los profesores de desempeñar su rol docente, que se concreta y se observa a través de su conducta diaria de clase (forma de interactuar con sus estudiantes, de explicar, evaluar, y ejercer la disciplina, etc.) (Díaz-Aguado, 1985; Doménech, 2004). Los estilos docentes afectan tanto a las acciones instructivas (actividades, pautas comunicativas, etc.) como a aquellas otras relativas a la organización y la vida del aula (organización de los espacios, los materiales y los tiempos, reglas y normas de funcionamiento, etc.). El pensamiento pedagógico del profesorado marca el devenir de las actuaciones docentes tanto en su dimensión más instruccional o de enseñanza, como en aquellas otras relativas a la gestión y la vida del aula (clima y ambiente de aprendizaje). Las creencias y las concepciones docentes varían en función del marco teórico que sobre la educación fundamenta la concepción de los componentes de la programación de aula/área y el tipo de relaciones que se dan entre ellos, incidiendo directa e indirectamente en la vida del aula: los papeles y la relación entre los sujetos del acto educativo, la intencionalidad educativa y la definición de los objetivos, el clima de aula y el ambiente de aprendizaje, la selección, priorización y organización de los contenidos, el sentido de la evaluación o la propuesta de actividades y su distribución temporal (Traver, 2004). Analizando los distintos elementos que caracterizan y definen los estilos de enseñanza y el pensamiento docente del profesorado, es fácil entrever la existencia de una fuerte relación entre ambos.

El pensamiento docente está, por tanto, en el origen y la génesis de los estilos de enseñanza. Pero, a su vez, depende de las explicaciones y creencias, de los marcos teórico-prácticos que sobre la educación manejan los docentes cuando diseñan, intervienen o valoran sus prácticas educativas. Como apunta Montero (1991), desde el paradigma mediacional centrado en el pensamiento del profesor habrá que hablar de estilos de enseñanza "cognitivamente orientados", que podemos encontrar englobados bajo el constructo teórico "perspectivas docentes". Constructo que, siguiendo a autores como Tabachnik y Zeichner (1985) incluye pensamientos y acción docentes en una misma categoría de estudio (citados por Montero, 1991, p. $294)^{2}$. Las perspectivas docentes parten de tesis comprensivas e integradoras, que intentan complementar y

\footnotetext{
${ }^{1}$ Este trabajo se ha realizado en el marco de un proyecto de investigación más amplio, financiado por UJI-Bancaixa (P11A2002-19), desarrollado durante el periodo comprendido entre 15/11/2002 y el 15/11/04.

${ }^{2}$ Para Tabachnik y Zeichner (1985) las perspectivas se concretan en "las direcciones en las que los profesores piensan acerca de su trabajo (por ej., metas, objetivos, concepciones de los alumnos, del currículum) y el grado en que esas creencias se corresponden con su acción en el aula" (p.2; cit. por Montero, 1991, p.294) .
} 
aunar los diferentes trabajos realizados a lo largo del tiempo bajo el epígrafe estilos de enseñanza ${ }^{3}$. De esta forma se reinterpretan los estilos de enseñanza desde la acción (comportamiento docente) y el pensamiento (creencias y concepciones docentes). La investigación educativa realizada desde el paradigma mediacional centrado en el profesor debe ser comprensiva, abordando de forma conjunta acciones y pensamiento (Clark y Peterson, 1989). Desde el análisis del comportamiento ponemos el énfasis en el análisis de las acciones instructivas y de gestión, desde el análisis del pensamiento en los procesos cognitivos que median en su comportamiento docente.

Podemos agrupar los marcos explicativos sobre la educación que manejan los docentes en dos grandes teorías explicativas del hecho educativo. Una nos sitúa en nuestra tradición pedagógica: los modelos transmisivos. La otra, nos acerca a los planteamientos defendidos por las últimas líneas de investigación en psicología y pedagogía: las teorías constructivas de la enseñanza y el aprendizaje. Mientras los planteamientos transmisivos se encuentran detrás de la mayoría de las prácticas actuales y están en la base de las preconcepciones docentes que las fundamentan, las propuestas de mejora educativa parecen caminar principalmente de la mano de las concepciones constructivas y de las concepciones interaccionistas de la educación (Traver, 2004). Una concepción transmisiva de la enseñanza-aprendizaje pone todo el peso de la educación en la figura del docente como modelo y fuente de educación, subordinando el aprendizaje a la enseñanza, siendo subsidiaria de una visión acumulativa del conocimiento que valora, de manera prioritaria, sus productos. Las teorías constructivas de la educación nacen de la concepción del alumno como un ser activo en referencia a los procesos intelectuales que pone en marcha en su aprendizaje, de manera que el peso de la educación recae en el otro polo: el aprendizaje. Si el conocimiento es fruto de una interacción entre sujeto y objeto, será esencialmente una construcción (Coll y Martí, 1991). Por tanto, valorará por encima de los productos los procesos de construcción de los aprendizajes. Mientras las visiones transmisivas centran sus explicaciones en uno de los dos polos de la educación, la enseñanza, y enfatizan el valor de los productos educativos, las visiones constructivistas se centran en el otro polo, el aprendizaje, y en el valor de los procesos.

Partiendo de trabajos previos de este equipo en los que se han caracterizado los distintos enfoques sobre las teorías psicopedagógicas que sustenta el profesorado, siguiendo a Doménech (1999c) podemos caracterizar estos dos marcos teóricos en cuatro teorías psicopedagógicas básicas en función de que el peso del análisis recaiga preferentemente en la enseñanza o el aprendizaje, el proceso o el producto (Tabla 1).

La caracterización de las teorías en estos cuatro enfoques nos permite analizar y clasificar de manera pormenorizada las perspectivas docentes del profesorado que ha participado en nuestra investigación. En función de ellos y a través del análisis de varias categorías descriptivas que, según hemos visto están detrás de las perspectivas docentes, hemos podido analizar elementos referenciales tanto de la acción (de enseñanza o instructiva y de gestión) como del pensamiento (creencias y concepciones docentes). Ahora bien, tanto en la clasificación realizada de las teorías psicopedagógicas como en la conceptualización de los dos marcos explicativos básicos del hecho educativo (transmisivos y constructivos) retratamos una realidad dicotómica que parece tensar los posicionamientos docentes a una u otra parte del

${ }^{3}$ Cabe recordar aquí el proceso evolutivo seguido por la investigación sobre estilos de enseñanza que abarca desde las primeras conceptualizaciones dicotómicas de carácter bastante especulativo, pasando por los estudios mucho más empíricos de Flanders y Bennett o los de Rosenshine y Stevens. 
péndulo. Aprendizaje o enseñanza, proceso o producto, docentes o alumnos, transmitir o construir, teoría o práctica. Posiblemente precisemos de nuevos marcos capaces de generar fórmulas de conciliación entre contrarios o, como dice Ferrés (2000), que utilicen lenguajes de síntesis acumulativa. Precisamos de nuevos enfoques que integren y concilien los elementos en tensión. Objetivo que rebasa la finalidad del presente artículo y que pensamos abordar en posteriores trabajos. Nuestra pretensión actual se enmarca, básicamente, en el contexto de estas notas preliminares: analizar y clasificar las perspectivas docentes desde tesis comprensivas e integradoras, en función de estos cuatros enfoques psicopedagógicos que dibujan la tensión real a la que se encuentran sometidas las situaciones educativas.

Tabla 1.

Caracterización de los cuatro tipos de creencias sobre la enseñanza/aprendizaje propuestos (Doménech, 1999a, 1999b, 2004)

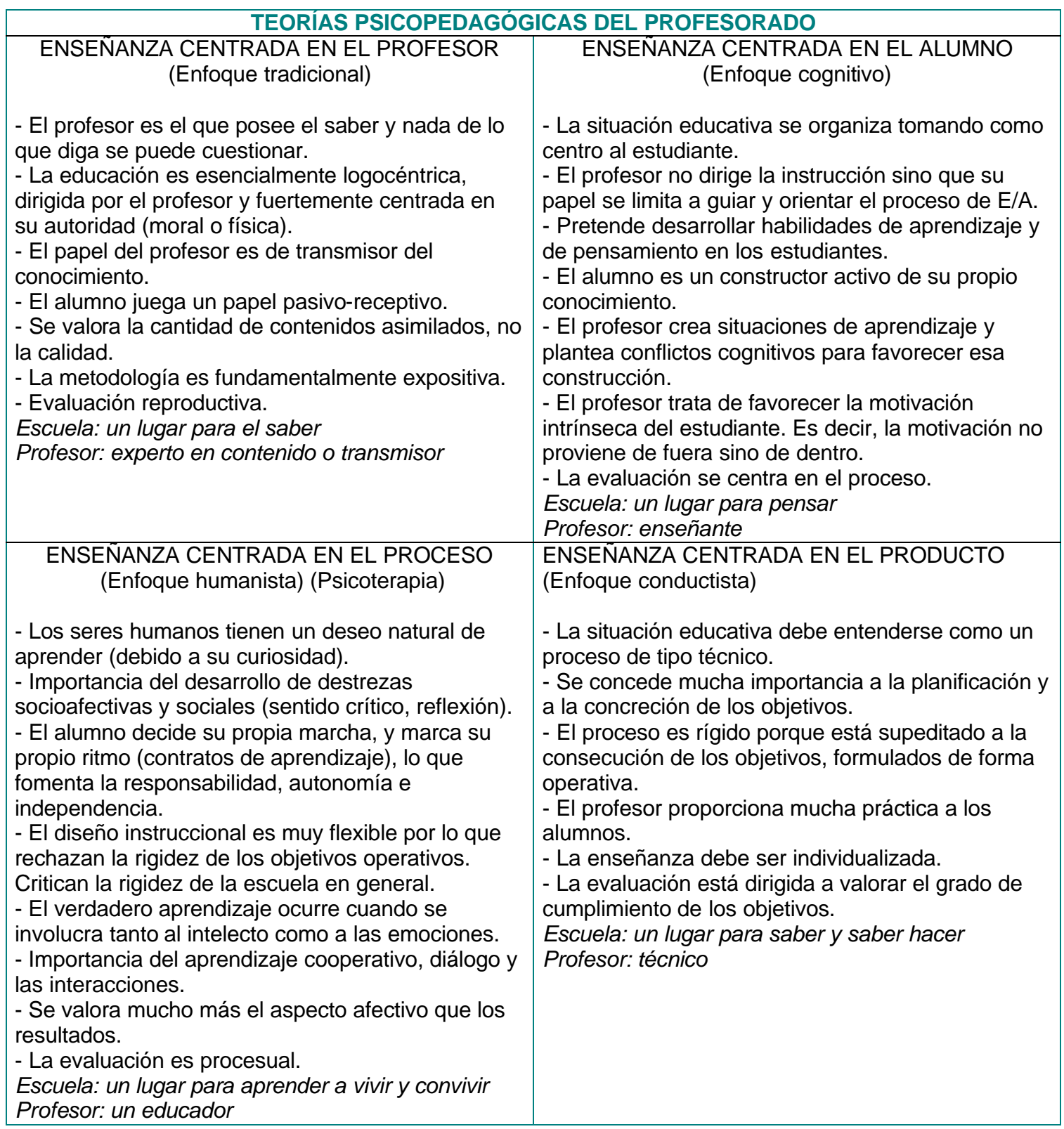

Las concepciones docentes pueden ser tanto fruto de la reflexión como de las presunciones y pueden actuar de forma consciente o inconsciente en su comportamiento en el aula. Los espacios de interactividad en los que se dan las experiencias de aprendizaje imprimen carácter de momentaneidad e imprevisión a las situaciones educativas, carácter que dificulta la actividad reflexiva del profesor. En la enseñanza interactiva las 
decisiones que tomamos están influidas por las impresiones que a nivel cognitivo y afectivo recibimos del aula, relegando a un segundo plano las estrategias reposadas de comparación de situaciones y elaboración de alternativas (Traver, 2004). En estas condiciones, toman fuerza las decisiones basadas en el sentido común, en el pensamiento vulgar-tradicional y precientífico. Sólo haciendo explícitas las presunciones docentes podemos estar en condiciones de cuestionarlas y empezar a generar pensamiento docente reflexivo para guiar nuestra acción educativa. Conocer y cuestionar el pensamiento docente de sentido común se convierte en el objetivo básico de nuestro trabajo de mejora educativa, por eso intentaremos tomar conciencia de la necesidad de una actitud reflexiva que cuestione lo obvio y nos permita realizar un "cambio didáctico" (Traver, 2004).

\subsection{La gestión social del aula}

La reflexión sobre las perspectivas docentes no puede realizarse en el vacío, sino que es necesario entenderlas desde un enfoque ecológico. De esta manera podemos indagar sobre las relaciones entre el ambiente de enseñanza-aprendizaje y la conducta y pensamiento docentes, en un vínculo de interacción, tal como señala Contreras(1990, en Angulo y Pérez, 1999:299): "Llevado a la vida del aula, el análisis ecológico de la enseñanza pretende dar cuenta de las acciones de profesores y alumnos en términos de las demandas del ambiente de clase. Para ello es fundamental conseguir establecer las características esenciales del ambiente, como marco de partida para entender las estrategias de actuación de profesores y alumnos."

En este sentido, el contexto inmediato al que nos remitimos es el aula, que podemos caracterizar a partir de las distintas conceptualizaciones que se han hecho sobre ella (Rué, 1998): un contexto relevante para la elaboración, por parte de los propios integrantes, de sus propias concepciones y prácticas de enseñanza y aprendizaje, a partir de las tareas realizadas, las experiencias y las interacciones vividas. Espacio educativo en el que todas las personas que lo ocupan desarrollan papeles de agente activo en la construcción de normas, en la reelaboración de las normas sociales, de los valores y en la construcción de reglas de relación social.

En este contexto tan peculiar, el grupo-clase establece sus relaciones y gestiona su vida cotidiana, por ello es importantísimo tener en cuenta la gestión social y educativa del aula (Fuégel, 2000:74): sus rutinas, los momentos de toma de decisiones en asamblea, la elaboración consensuada de las normas de clase, el reparto de responsabilidades y cargos, los debates y la resolución de conflictos, el trabajo en grupo y el clima afectivo para el aprendizaje y la convivencia, la acogida a nuevos miembros, la participación de la comunidad, etc. (Varios, 2003).

Si partimos de una concepción de la educación como proceso de comunicación, cuyo objeto es la creación compartida del conocimiento, podemos analizar cómo se produce la comunicación entre el alumnado y el profesorado, revisando el discurso y el contexto educativos, así como otros aspectos que configurarán la enseñanza y el aprendizaje en el aula, y así poder entender de qué forma y en qué condiciones se pueden desarrollar unas perspectivas mutuas por los participantes en el acto educativo: desde el punto de vista del discurso educativo como proceso de comunicación e interacción social, hemos de señalar la importancia que toma la explicitación de las reglas básicas educacionales, reglas implícitas del habla y las prácticas educativas. La no explicitación o reconocimiento de las mismas puede dar lugar a malentendidos que obstaculizarán el proceso de enseñanza-aprendizaje. Por su parte, el discurso en el aula dependerá, para ser inteligible, de la acumulación gradual de contextos compartidos de habla y experiencia. 
Por tanto, consideramos que la explicitación de las reglas y normas de funcionamiento del aula son un factor clave para la gestión social del espacio de clase, de tal manera que, como señala Cruz Pérez (1996:225): "es fundamental que las normas sean negociadas y consensuadas con los alumnos y, a ser posible, elaboradas por procedimientos de participación democrática entre alumnos y profesores. Ello otorga a las normas una fuerza moral que facilita su aceptación y cumplimiento, además del valor educativo del proceso y su contribución a la formación de personalidades autónomas".

Por otro lado, la gestión social del aula también comporta la gestión del curriculum en la misma, creando un clima de aula y ambiente de aprendizaje determinado a través de la organización del grupoclase, de los espacios y los tiempos escolares básicamente. Mientras las reglas y normas de funcionamiento vienen caracterizadas por la dinámica de trabajo y por los papeles a desarrollar por parte del profesorado y del alumnado, éstos -a su vez- dependen y están matizados en la práctica, en función de las características definitorias del ambiente de aprendizaje utilizado para realizar la experiencia educativa.

En la organización del grupo-clase, la diversificación de los agrupamientos y su uso estratégico nos permiten una mejor gestión del clima y ambiente de aprendizaje (Sales Ciges, 2004). En este sentido, también a través del tipo de espacio, de la ubicación del mobiliario podemos deducir el tipo de comunicación que se establece en el aula, estableciéndose toda una dialéctica entre el espacio vital del sujeto y el espacio de que se apropia. El uso del espacio y del tiempo escolar como recursos didácticos se debe a su influencia en la complejidad del curriculum, la racionalidad didáctica, los nuevos recursos didácticos, la utilización del entorno y la organización de las personas en el centro (Doménech y Viñas, 1997).

Por último apuntar que cuando el grupo-clase se organiza de forma flexible y diversificada en sus actividades, la utilización de espacios y materiales, la distribución del tiempo en el aula y en el centro también deben responder a esta flexibilidad y apertura, para que las situaciones que se den sean más vivas y dinámicas (Uría, 1998). Todavía hoy la organización de los horarios se basa en criterios poco pedagógicos, desde una visión monocrónica del tiempo para la jornada tanto de los estudiantes, del profesorado y de la comunidad en general (visión lineal, predeterminada, insensible al contexto, técnica) (Sales Ciges, 2004). Esta concepción del tiempo aumenta la rigidez, dificulta las relaciones interpersonales, no facilita la colegialidad, no contribuye a la interdisciplinariedad y no permite atender a la diversidad. Sería, pues, necesario primar una visión policrónica del tiempo que refleje la complejidad, densidad e intensidad de las tareas y relaciones interpersonales en el contexto escolar (Martín Bris, Margalef y Rayón, 2000).

\section{ESTUDIO EMPÍRICO. METODOLOGÍA}

\subsection{Muestra y Procedimiento}

Basándonos en otros estudios previos, señalados en la fundamentación teórica anterior, nuestra hipótesis de partida consiste en afirmar que debe existir una relación directa entre las acciones instructivas y el tipo de gestión del aula, y de éstas con las creencias psicopedagógicas que sustentan su pensamiento docente. La confluencia de estos tres factores, correspondientes a los ámbitos de la acción y el pensamiento docente, desvelará las diferentes perspectivas docentes del profesorado de secundaria de la muestra objeto de estudio, entendiendo las perspectivas docentes como estilos de enseñanza cognitivamente orientados (Montero, 1991).

Podemos concretar los objetivos de la presente investigación de la siguiente forma: 
- Definir y configurar las perspectivas docentes desde tesis comprensivas que abarcan tanto el campo del pensamiento docente (creencias) como el de la acción (tareas instructivas y de gestión).

- Identificar y examinar las perspectivas docentes presentes en la muestra de profesores de Secundaria estudiados, en base a las dimensiones anteriormente propuestas, consideradas de forma integrada (creencias, tareas instructivas y de gestión).

La muestra estaba compuesta por 249 profesores de secundaria (130 hombres y 117 mujeres) de distintas materias, todos ellos pertenecientes a 21 centros educativos, públicos y privados, de la provincia de Castellón (España). Los centros fueron seleccionados de forma aleatoria (en caso de existir más de un centro) de entre los municipios pertenecientes a las comarcas de la Plana Baixa y de la Plana Alta de la provincia de Castellón. La edad de los profesores estaba comprendida entre 20 y 50 años (el $11 \%$ de la muestra tenían entre $20-29$ años, el $26.3 \%$ entre $30-39$ años, el $43.0 \%$ entre $40-50$ años y el $9.6 \%$ tenían más de 50 años de edad). Los profesores participantes respondieron, durante el curso 03-04, de forma anónima y voluntaria a una batería de test en el contexto de un proyecto de investigación más amplio, pero en el presente trabajo sólo se han presentado los instrumentos utilizados en esta investigación.

A continuación pasamos a señalar y comentar los instrumentos de medida, o cuestionarios, utilizados en la presente investigación:

- Cuestionario sobre "Creencias psicopedagógicas del profesorado sobre el proceso de enseñanza-aprendizaje", de Doménech (1999; 2004).

- Cuestionario "Registro de la acción docente del profesor", elaborado por nosotros para este estudio.

El primero de ellos, elaborado por Doménech (1999; 2004) estaba compuesto inicialmente por 56 ítems de 6 escalares puntuados de la siguiente forma: 6 . Totalmente de acuerdo, 5. Mayoritariamente de acuerdo, 4. Más de acuerdo que en desacuerdo, 3. Más en desacuerdo que de acuerdo, 2. Mayoritariamente en desacuerdo, 1. Totalmente en desacuerdo. Este cuestionario ha permitido la clasificación de las concepciones del profesor de secundaria sobre la enseñanza y el aprendizaje en dos dimensiones bipolares: profesor-alumno y proceso-producto, que se corresponden con los cuatro paradigmas instructivos clásicos: paradigma tradicional (enfoque centrado en el profesor), paradigma conductista/tecnológico (enfoque centrado en el producto), paradigma cognitivo/constructivista (enfoque centrado en el alumno) y paradigma humanista/ecológico (enfoque centrado en el proceso). Este planteamiento permite definir de forma clara y operativa el perfil de las creencias psicopedagógicas del profesor en torno a dos ejes bipolares opuestos, que gráficamente se muestran cruzados ortogonalmente y delimitados por cada uno de los enfoques considerados. Este cuestionario ha mostrado una consistencia interna aceptable en las dos subescalas bipolares, profesor-alumno y proceso-producto.

El segundo cuestionario utilizado (Registro de la acción docente) está compuesto por un total de 46 variables valoradas según una escala que oscila entre los siguientes valores: 1. Raramente o nunca; 2 . Con poca frecuencia; 3. Con mucha frecuencia. Este cuestionario está estructurado en dos subescalas que recogen las principales variables que configuran la acción docente tanto en su dimensión más instructiva como de gestión del aula. Así, en la primera subescala: A (puntos 1 al 7) aparecen las variables referidas a 
las tareas instructivas agrupadas según 7 dimensiones: tareas, agrupamientos, actividades, materiales de apoyo, refuerzos, técnicas didácticas y técnicas de evaluación. En la segunda subescala: B (punto 8) aparecen relacionadas las variables relacionadas con las tareas de gestión, referidas a reglas y normas de funcionamiento en el aula, agrupadas en cuatro dimensiones: proceso de aprendizaje, interacciones en el aula, rutinas y uso de materiales y recursos didácticos. Este cuestionario ha mostrado para las dos subescalas una consistencia interna satisfactoria aunque moderada ( $\alpha$ de Cronbach $=0.62$ y 0.59 respectivamente).

\subsection{Resultados}

Para realizar el tratamiento estadístico de los datos de la muestra se utilizó el paquete estadístico SPSS (Norusis, 1999). En primer lugar se trató de determinar la validez de constructo a través de sucesivos análisis factoriales, así como la consistencia interna de los instrumentos utilizados. Posteriormente, se realizó un análisis correlacional de los componentes extraídos en los factoriales, con el fin de determinar el nivel de relación entre dichos componentes.

Análisis factoriales y descriptivos de las escalas utilizadas

Cuestionario creencias psicopedagógicas: Después de adaptarlo y aplicarlo a la muestra objeto de estudio se realizó un análisis factorial de componentes principales, con rotación varimax, para determianr su validez de constructo. También se calculó el alpha de Cronbach para determinar su fiabilidad. La tabla 3 muestra los detalles del factorial obtenido, así como los descriptivos de cada componente extraído.

Tabla 3:

Matriz de componentes rotados para las dos dimensiones del cuestionario de creencias, índices de fiabilidad y descriptivos de los factores obtenidos para las dos dimensiones del cuestionario de creencias del profesor. $(n=239)$

\begin{tabular}{|c|c|c|c|c|c|}
\hline \multicolumn{3}{|c|}{ Dimensión Profesor-Alumno } & \multicolumn{3}{|c|}{ Dimensión Proceso-Producto } \\
\hline & $\begin{array}{l}2 \text { Factores } \\
\text { C. Profesor }\end{array}$ & $\begin{array}{l}\text { extraídos } \\
\text { C. Alumno }\end{array}$ & & $\begin{array}{r}2 \text { Factores e } \\
\text { C. Proces } \\
\text { Produ }\end{array}$ & $\begin{array}{l}\text { extraídos } \\
\text { so C. } \\
\text { cto }\end{array}$ \\
\hline CRIT1 & .673 & $8.298 \mathrm{E}-02$ & CRIT7 & $\begin{array}{r}.716 \\
\end{array}$ & $-4.813 E-02$ \\
\hline CRIT13 & .644 & $-1.431 \mathrm{E}-02$ & CRIT43 & $.664-$ & $-4.664 \mathrm{E}-02$ \\
\hline CRIT9 & .617 & -.251 & CRIT11 & .626 & .135 \\
\hline CRIT29 & .588 & -.173 & CRIT31 & .611 & -.117 \\
\hline CRIT49 & .560 & -.143 & CRIT15 & .584 & -.193 \\
\hline CRIT53 & .540 & -.247 & CRIT47 & .568 & -.216 \\
\hline CRIT2 & $-2.891 \mathrm{E}-02$ & .667 & CRIT51 & .454 & -.428 \\
\hline CRIT10 & 6.609E-02 & .658 & CRIT32 & -.231 & .691 \\
\hline CRIT14 & -7.685E-02 & .637 & CRIT41 & 5.233E-02 & .627 \\
\hline CRIT50 & -.310 & .584 & CRIT36 & 8.921E-02 & .612 \\
\hline CRIT18 & -.282 & .562 & CRIT4 & $-3.313 \mathrm{E}-02$ & .589 \\
\hline \multirow[t]{2}{*}{ CRIT34 } & -.227 & .497 & CRIT12 & -.236 & .495 \\
\hline & & & CRIT24 & -.209 & .464 \\
\hline Varianza & $27.53 \%$ & $12.52 \%$ & & $25.90 \%$ & $13.40 \%$ \\
\hline Medias & 3,45 & 4,42 & & 4,12 & 3,18 \\
\hline D.T. & 0,79 & 0,65 & & 0,68 & 0,71 \\
\hline Cronbach & 0.69 & 0.67 & & 0.71 & 0.65 \\
\hline
\end{tabular}

Subescala dimensión Profesor-alumno (12 items). Se obtuvieron 2 factores: Factor 1 centrado en el profesor (Ejemplos de items de este factor: "El conocimiento de los contenidos del área/asignatura es el requisito más importante para ser un buen profesor", "La cantidad de conocimientos asimilados por el alumno durante su aprendizaje nos indica el progreso alcanzado", etc.). Factor 2 centrado en el alumno (Ejemplos de items de este factor: "Ver las cosas desde el punto de vista del estudiante es la clave para que 
obtengan un buen rendimiento escolar", "Antes de tomar decisiones que afecten al grupo-clase, el profesor de secundaria debería contar con la opinión de los alumnos", etc.).

Subescala dimensión Proceso-producto (13 items). Se obtuvieron 2 factores: Factor 1 centrado en el proceso (Ejemplos de items de este factor: "El grado de satisfacción de los alumnos es un indicador importante de la calidad del proceso instruccional", "Desarrollar en el alumno la pasión por saber y aprender debería anteponerse a la obsesión por el rendimiento y los resultados académicos, etc.). Factor 2 centrado en el producto (Ejemplos de items de este factor: "Considero prioritario terminar de impartir el programa de la asignatura que se había planificado al principio de curso", "El control/examen es la mejor forma de conocer el progreso del alumno", etc.).

Cuestionario acción docente: Este cuestionario esta formado por dos subescalas. De la misma forma que hicimos con el cuestionario anterior, se determinó la validez de constructo y fiabilidad de cada una de ellas. Los componentes extraídos a través del análisis factorial aplicado a cada subescala, los descriptivos de cada componente extraído y la consistencia interna se pueden observar en las tablas 4 y 5 .

Tabla 4

Matriz de componentes rotados del cuestionario de acciones docentes, índices de fiabilidad y descriptivos de los factores obtenidos, referentes al del Factorial del cuestionario de acciones docentes. Matriz de componentes rotados

\begin{tabular}{|c|c|c|c|c|c|}
\hline & \multicolumn{5}{|c|}{ Componente } \\
\hline & 1 & 2 & 3 & 4 & 5 \\
\hline estit13 & ,815 &,- 001 & ,039 & ,003 &,- 010 \\
\hline estit62 & ,796 & ,041 & 144 & ,042 &,- 015 \\
\hline estit23 & ,594 & ,318 & ,040 &,- 017 &,- 362 \\
\hline estit75 & ,550 &,- 051 & 270 &,- 238 & 079 \\
\hline estit32 & ,527 & ,053 & , 179 &,- 101 & , 181 \\
\hline estit34 & ,031 & ,778 & , 123 &,- 007 & ,057 \\
\hline estit63 &,- 059 & 675 &,- 044 &,- 133 & , 120 \\
\hline estit35 & ,177 & ,656 & ,068 &, 146 & ,018 \\
\hline estit43 & ,148 & ,032 & ,759 &,- 105 & ,083 \\
\hline estit24 &, 141 & 175 & ,730 &,- 022 &,- 138 \\
\hline estit73 & 185 &,- 026 & ,539 & 193 &,- 066 \\
\hline estit61 &,- 019 &,- 143 &,- 008 & ,834 &, 162 \\
\hline estit11 &,- 156 & ,137 & ,052 & 805 & ,069 \\
\hline estit41 & ,099 &,- 115 &,- 077 & 214 & ,718 \\
\hline estit33 &,- 122 & ,194 & ,143 & ,049 & 642 \\
\hline estit74 &, 137 & ,226 &,- 285 &,- 021 & ,572 \\
\hline Varianza & $15,07 \%$ & $11,07 \%$ & $10,37 \%$ & $9,67 \%$ & $9,40 \%$ \\
\hline Media & 2,01 & 2,43 & 1,93 & 2,82 & 2,78 \\
\hline D.T. & 0,50 & 0,51 & 0,57 & 0,35 & 0,36 \\
\hline Cronbach & 0.72 & 0.57 & 0.54 & 0.65 & 0.46 \\
\hline
\end{tabular}

estil 1 (it. 13,32,75,62,23) $\rightarrow$ Tareas abiertas-orientadas

estil2 (it. 34, 63, 35) $\rightarrow$ Trabajo dirigido

estil3 (it. 43,24,73) $\rightarrow$ Trabajo con grupo-clase

estil4 (it. 61,11) $\rightarrow$ Enseñanza expositiva

estil5 (it. 41,33, 74) $\rightarrow$ Tareas cerradas-obligatorias 
Tabla 5.

Gestión. Matriz de componentes rotados del cuestionario de gestión, índices de fiabilidad y descriptivos de los factores obtenidos. Matriz de componentes rotados

\begin{tabular}{|c|c|c|c|c|}
\hline & \multicolumn{4}{|c|}{ Componente } \\
\hline & 1 & 2 & 3 & 4 \\
\hline est80cc & ,874 &, 017 &,- 069 &, 015 \\
\hline est80dc & 873 & ,047 & ,009 & ,025 \\
\hline est80bc & 856 &,- 070 & ,085 & ,098 \\
\hline est80ac & ,805 &,- 033 & ,029 & ,054 \\
\hline est80cd &,- 030 & ,838 & , 153 &,- 028 \\
\hline est80dd & ,020 & 818 & ,092 &,- 065 \\
\hline est80bd &,- 017 & 806 & , 129 &,- 083 \\
\hline est80ad &,- 013 & ,773 & ,036 & ,053 \\
\hline est80bb & ,014 & ,061 & 844 &,- 111 \\
\hline est80cb &,- 007 & ,050 & 798 &,- 160 \\
\hline est80ab & ,007 & ,223 & 796 &,- 122 \\
\hline est80db & ,040 & 157 & ,576 &,- 316 \\
\hline est80da & , 142 &,- 090 & ,008 & ,832 \\
\hline est80ca & ,075 &,- 065 &,- 239 & ,794 \\
\hline est80ba & ,010 & ,059 &,- 250 & 698 \\
\hline est80aa &,- 038 &,- 009 &,- 425 &, 523 \\
\hline Varianza & $18,58 \%$ & $17,52 \%$ & $16,80 \%$ & $13,54 \%$ \\
\hline Medias & 2,60 & 1,34 & 1,88 & 2,29 \\
\hline D.T. & 0,57 & 0,50 & 0,67 & 0,59 \\
\hline $\begin{array}{l}\text { Cronbac } \\
\text { h }\end{array}$ & 0,88 & 0,64 & 0,80 & 0,75 \\
\hline
\end{tabular}

Componente $1 \rightarrow$ gestión c (explícita)

Componente $2 \rightarrow$ gestión d (implícita)
Componente $3 \rightarrow$ gestión b (autocrática)

Componente $4 \rightarrow$ gestión a (negociada)

Subescala A. Tareas instructivas (16 ítems). Se obtuvieron 5 factores: Factor 1: Abiertas-orientadas. (Ejemplos de Ítems de este factor: (23) Trabajo en pequeños grupos: aprendizaje cooperativo, realización de proyectos); (32) Actividades Optativas; (75) Evaluación a partir de elaboración de tareas: trabajos, informes, redacciones, etc. (62) Método de proyectos y realización de investigaciones por los alumnos); (13) Realización de proyectos de trabajo e investigación. Factor 2: Trabajo dirigido. (Ejemplos de items de este factor: (34) Actividades dirigidas; (35) Actividades semidirigidas; (63) Trabajo guiado). Factor 3: Trabajo con grupo clase. (Ejemplos de Ítems de este factor: (24) Trabajo en grupo-clase: debate, explicación, proyección de audiovisuales; (43) materiales audiovisuales: retroproyector, vídeo, diapositivas, casete... como materiales de apoyo; (73) Evaluación a través de preguntas de ensayo o desarrollo libre. Factor 4: Enseñanza expositiva. (Ejemplos de Ítems de este factor: (61) Método expositivo; (11) Explicación). Factor 5: Actividades cerradas-obligatorias (Ejemplos de ítems de este factor: (33) Actividades obligatorias (74) Evaluación a partir de ejercicios y problemas de aplicación de conceptos; (41) Pizarra).

Subescala B. Tareas de gestión (16 ítems). Se obtuvieron 4 factores: Factor 1: Gestión explícita (Ejemplos de items de este factor: "Regular el proceso de aprendizaje de forma explícita", "Regular las interacciones del aula entre profesor-alumnos y alumnos entre sí de forma explícita”, etc.) Factor 2: Gestión implícita (Ejemplos de items de este factor: "Regular el proceso de aprendizaje de forma implícita", "Regular las rutinas de forma implícita", etc.). Factor 3: Gestión autocrática (Ejemplos de items de este factor: "Regular el uso de materiales y recursos didácticos de forma autocrática", "Regular las rutinas de forma autocrática", etc.) Factor 4: Gestión negociada (Ejemplos de items de este factor: "Regular el proceso de 
aprendizaje de forma negociada", "Regular las interacciones del aula entre profesor-alumnos y alumnos entre sí de forma negociada", etc.).

\section{Tratamiento factorial conjunto de los dos cuestionarios}

Se realizó un análisis factorial conjunto de los dos cuestionarios utilizados en el estudio para determinar la configuración estructural en base a las creencias, tareas instructivas y de gestión, tratando conjuntamente la acción y pensamiento docente. Los resultados obtenidos del análisis factorial realizado, así como los descriptivos de los componentes extraídos, se muestran en la tabla 6.

Tabla 6

Factorial conjunto de los tres componentes (creencias, acciones instructivas y gestión del aula)

Matriz de componentes rotados

\begin{tabular}{|c|c|c|c|c|}
\hline & \multicolumn{4}{|c|}{ Componente } \\
\hline & 1 & 2 & 3 & 4 \\
\hline $\begin{array}{l}\text { c-proces (it. } \\
7,43,11,31,15,47,51 \text { ) }\end{array}$ & ,868 &,- 007 &,- 039 &,- 091 \\
\hline $\begin{array}{l}\text { c-alumno (it. } \\
2,10,14,50,18,34 \text { ) }\end{array}$ & ,854 &,- 081 &,- 084 &,- 038 \\
\hline $\begin{array}{l}\text { c-profe (it. } \\
1,13,9,29,49,53 \text { ) }\end{array}$ &,- 617 &,- 286 & , 188 & ,414 \\
\hline $\begin{array}{l}\text { c-produc (it. } \\
41,36,4,12,24) \\
\text { it } 43,24,73\end{array}$ &,- 505 &,- 202 & ,020 & ,464 \\
\hline $\begin{array}{l}\text { Trabajo con grupo- } \\
\text { clase } \\
\text { it } 13,62,23,75,32\end{array}$ &,- 005 & ,731 &,- 102 &,- 182 \\
\hline $\begin{array}{l}\text { Tareas abiertas- } \\
\text { orientadas }\end{array}$ & ,098 & ,712 & ,058 &,- 200 \\
\hline explicita &,- 071 & ,554 &,- 075 & ,215 \\
\hline $\begin{array}{l}\text { it } 34,63,35 \\
\text { Trabajo dirigido }\end{array}$ & , 193 & ,525 & ,277 & ,294 \\
\hline autocratica &,- 232 &,- 040 & ,810 & ,051 \\
\hline negociada & 259 & 228 &,- 696 & 214 \\
\hline $\begin{array}{l}\text { implicita } \\
\text { it } 41,33,74\end{array}$ & ,208 & ,203 & ,662 & , 149 \\
\hline $\begin{array}{l}\text { Tareas cerradas- } \\
\text { obligatorias }\end{array}$ &,- 023 & ,141 &,- 003 & ,737 \\
\hline $\begin{array}{l}\text { it } 61,11 \\
\text { Enseñanza expositiva }\end{array}$ &,- 202 &,- 102 & ,013 & ,565 \\
\hline Varianza & $18,27 \%$ & $14,44 \%$ & $13,22 \%$ & $11,80 \%$ \\
\hline
\end{tabular}

Se obtuvieron 4 componentes. Factor 1: Constructivista. Aglutina, principalmente, los items relativos a los siguientes componentes: creencias centradas en el alumno y creencias centradas en el proceso. Factor 2: Transición. Aglutina, principalmente, los items relativos a los siguientes componentes: tareas de instrucción de tipo trabajo en grupo clase, actividades abiertas-orientadas y trabajo dirigido, y tareas de gestión explícita. Factor 3: Gestión. Aglutina, principalmente, los items relativos a los siguientes componentes: tareas de gestión autocrática e implícita. Factor 4: Transmisivo. Aglutina, principalmente, los items relativos a los siguientes componentes: creencias centradas en el producto, creencias centradas en el profesor y tareas instructivas de tipo directivo, cerradas-obligatorias y enseñanza expositiva.

\section{Análisis correlacional}

Calculamos las correlaciones entre los componentes obtenidos de los analisis factoriales realizados en cada uno de los cuestionarios (ver tabla 8). 
Centrándonos en la categoría del pensamiento docente $\mathrm{y}$, en concreto, en las creencias psicopedagógicas de los mismos encontramos que: el modelo de enseñanza/aprendizaje centrado en el profesor (C.Profe) correlaciona de forma negativa y significativa $(\tilde{a}=-.418, p<.01)$ con el modelo de enseñanza/aprendizaje centrado en el alumno (C.Alumno); y el modelo de enseñanza/aprendizaje centrado en el proceso (C.Proces) correlaciona de forma negativa y significativa ( $\tilde{a}=-368, p<.01$ ) con el modelo de enseñanza/aprendizaje centrado en el producto (C.Produc). Por otra parte, el modelo de enseñanza/aprendizaje centrado en el profesor (C.Profe) correlaciona de forma positiva y estadísticamente significativa ( $\tilde{\mathrm{a}}=.649, \mathrm{p}<.01$ ) con el modelo de enseñanza/aprendizaje centrado en el producto (C.Produc); y el modelo de enseñanza/aprendizaje centrado en el proceso (C.Proces) correlaciona de forma positiva y estadísticamente significativa ( $\tilde{a}=.691, \mathrm{p}<.01$ ) con el modelo de enseñanza/aprendizaje centrado en el alumno (C.Alumno). Basándonos en las intercorrelaciones obtenidas entre los modelos de enseñanza/aprendizaje se observa una tendencia bipolar manifestada por los siguientes núcleos: modelos centrados en profesor-producto y modelos centrados en alumno-proceso. Esta forma de categorizar las concepciones de los profesores ha sido defendida por otros investigadores como Kember (1997) que clasifica las creencias del profesor en dos dimensiones similares: centradas en el profesor/contenido versus centradas en el estudiante/aprendizaje. Tratando de resumir y apoyados por los resultados del análisis correlacional, se agrupó la muestra en dos categorías (profesores centrados predominantemente en creencias profesor-producto versus centrados en creencias alumno-proceso) según si la puntuación obtenida era mayor en una o en otra categoría. De esta forma obtuvimos dos grupos o submuestras. Una submuestra de 51 sujetos que puntuaron más alto en la categorías profesor-producto y una submuestra de 161 sujeto que puntuaron más alto en la categoría alumno-proceso.

Centrándonos en la categoría de la acción docente y, en concreto, en las tareas instructivas encontramos que: las tareas de instrucción de tipo abiertas-orientadas correlaciona de forma positiva y estadísticamente significativa con las de tipo trabajo con grupo-clase ( $\tilde{a}=.362, p<.01)$ y las de tipo dirigidas $(\tilde{a}=.170, p<.05)$, y de forma negativa y estadísticamente significativa con las de tipo expositiva $(\tilde{a}=-.139$, $\mathrm{p}<.05)$. Por el contrario, las tareas instructivas de tipo dirigido correlaciona de forma positiva $y$ estadísticamente significativa con las de tipo abiertas-orientadas ( $\tilde{a}=.170, p<.05)$, con las de tipo trabajo con grupo-clase $(\tilde{a}=.148, p<.05)$ y con las de tipo cerradas-obligatorias $(\tilde{a}=.214, p<.01)$; mientras que las tareas de tipo grupo-clase correlacionan de forma positiva y estadísticamente significativa con las de tipo abiertasorientadas $(\tilde{a}=.362, p<.01)$ y las de tipo trabajo dirigido $(\tilde{a}=.148, p<.05)$. Las tareas instructivas de tipo enseñanza expositiva correlacionan de forma positiva y estadísticamente significativa con las de tipo cerradas-obligatorias $(\tilde{a}=.178, p<.01)$ y de forma negativa y estadísticamente significativa con las de tipo abiertas-orientadas $(\tilde{a}=-.139, \mathrm{p}<.05)$. Finalmente, las tareas instructivas de tipo cerradas-obligatorias correlacionan de forma positiva y estadísticamente significativa con las de tipo trabajo dirigido ( $\tilde{a}=.214$, $\mathrm{p}<.05)$ y con las de tipo expositivo ( $\tilde{\mathrm{a}}=.178, \mathrm{p}<.01)$. De la tabla de correlaciones se desprende un cierto posicionamiento bipolar entre el núcleo abiertas-orientadas/trabajo con grupo-clase (característico de los enfoques constructivistas) y el núcleo cerradas-obligatorias/expositivo (característico de los enfoques transmisivos); mientras que las de tipo dirigido mantienen una correlación estadísticamente significativa con alguno de los componentes de cada uno de los dos polos.

Centrándonos en la categoría de la acción docente y, en concreto, en las tareas de gestión encontramos que: las tareas de gestión de tipo negociada correlacionan de forma negativa y 
estadísticamente significativa con las de tipo autocrática ( $\tilde{\mathrm{a}}=-.472, \mathrm{p}<.01)$ y mantienen una tendencia negativa con las de tipo implícita ( $\tilde{a}=-.118$ ); las tareas de gestión de tipo autocrática correlacionan de forma positiva y estadísticamente significativa con las de tipo implícita ( $\tilde{a}=.285, p<.01$ ) y de forma negativa y estadísticamente significativa con las de tipo negociada ( $\tilde{a}=-.472, p<.01$ ). Las tareas de gestión de tipo explícita no presentan correlaciones estadísticamente significativas con ninguno de los otros tipos, tan sólo se observa una tendencia negativa con las de tipo implícita (ã = -.071). Finalmente las tareas de gestión de tipo implícita correlacionan de forma positiva y estadísticamente significativa con las de tipo autocrática (ã $=$ .285, $\mathrm{p}<.01$ ) y marcan una tendencia negativa en la correlación con las de tipo explícita ( $\tilde{a}=-.071$ ) y las de tipo negociada ( $\tilde{a}=-.118$ ). De forma general se aprecia también un cierto posicionamiento bipolar entre el núcleo negociada-explícita y el núcleo autocrática-implícita.

Centrándonos en la categoría de las perspectivas docentes y analizando de forma conjunta pensamiento y acción docente encontramos que: se observa que las creencias centradas en el profesor y en el producto correlacionan de forma negativa y estadísticamente significativa con las tareas instructivas de tipo abiertas-orientadas ( $\tilde{a}=-.286, p<.01, \tilde{a}=-.197, p<.01$ respectivamente), con las de tipo trabajo con grupo-clase (ã $=-.218, p<.01, \tilde{a}=-.146, p<.05$ respectivamente) y con las tareas de gestión tipo negociada ( $\tilde{a}=-.196, p<.01, \tilde{a}=-.134$, respectivamente) , y positiva y estadísticamente significativa con las instructivas de tipo expositivo ( $\tilde{a}=.251, \mathrm{p}<.01, \tilde{a}=.213, p<.01$ respectivamente) y con las tareas de gestión de tipo autocrática ( $\tilde{a}=.277, \mathrm{p}<.01, \tilde{a}=.085$ respectivamente). Por el contrario, las creencias centradas en el alumno $y$ en el proceso correlacionan de forma positiva y estadísticamente significativa con las tareas instructivas de tipo abiertas-orientadas ( $\tilde{a}=.139, \mathrm{p}<.05, \tilde{a}=.126$ respectivamente) y con las tareas de gestión de tipo negociada ( $\tilde{a}=.186, p<.01, \tilde{a}=.252, p<.01$ respectivamente), y negativa y estadísticamente significativa con las tareas instructivas de tipo expositivo ( $\tilde{a}=-.206, p<.01, \tilde{a}=-.187, p<.01$ respectivamente) y las de tipo cerradas-obligatorias (ã $=-.124, \tilde{a}=-.186, p<.01$ respectivamente) y con las tareas de gestión autocráticas (ã $=-.288, \mathrm{p}<.01, \tilde{a}=-.219, \mathrm{p}<.01$ respectivamente). De forma global, podemos apuntar que se aprecia un cierto posicionamiento bipolar conformado por dos núcleos relacionales pensamiento-acción docente y que podemos caracterizar en función de las perspectivas docentes dominantes(tabla 7):

Tabla 7.

Caracterización de las perspectivas docentes en función de los principales componentes del pensamiento y la acción docente

\begin{tabular}{|c|c|c|c|}
\hline Perspectiva docente & Creencias & Tareas instructivas & Tareas de gestión \\
\hline Transmisiva & $\begin{array}{c}\text { Centradas en el profesor } \\
\text { y en el producto }\end{array}$ & De tipo expositivo & De tipo autocrática \\
\hline Constructiva & $\begin{array}{c}\text { Centradas en el alumno } \\
\text { y en el proceso }\end{array}$ & $\begin{array}{c}\text { De tipo abiertas- } \\
\text { orientadas }\end{array}$ & De tipo negociada \\
\hline
\end{tabular}


Tabla 8

Correlaciones con todos los factores procedentes de las escalas y subescalas utilizadas (creencias, tareas instruccionales y gestión)

\begin{tabular}{|c|c|c|c|c|c|c|c|c|c|c|c|c|c|}
\hline & $\begin{array}{c}\begin{array}{c}\text { c-profe } \\
\text { (it. }\end{array} \\
1,13,9,2 \\
9,49,53) \\
\end{array}$ & $\begin{array}{c}\begin{array}{c}\text { C- } \\
\text { alumno } \\
\text { (it. }\end{array} \\
2,10,14, \\
50,18,34 \\
\text { ) }\end{array}$ & $\begin{array}{c}\text { c-proces } \\
\text { (it. } \\
7,43,11, \\
31,15,47 \\
, 51) \\
\end{array}$ & $\begin{array}{c}\text { c-produc } \\
\text { (it. } \\
41,36,4, \\
12,24) \\
\end{array}$ & $\begin{array}{c}\text { Tareas } \\
\text { abiertas- } \\
\text { orientad } \\
\text { as it } \\
13,62,23 \\
, 75,32 \\
\end{array}$ & $\begin{array}{c}\text { Trabajo } \\
\text { dirigido } \\
\text { it } 34,63,35\end{array}$ & $\begin{array}{c}\text { Trabajo } \\
\text { con grupo- } \\
\text { clase it } \\
43,24,73 \\
\end{array}$ & $\begin{array}{c}\text { Enseñanz } \\
\text { a } \\
\text { expositiva } \\
\text { it } 61,11 \\
\end{array}$ & $\begin{array}{c}\text { Tareas } \\
\text { cerradas- } \\
\text { obligatoria } \\
\text { s it } \\
41,33,74 \\
\end{array}$ & negociada & autocratica & explicita & implicita \\
\hline $\begin{array}{l}\text { c-profe (it. } \\
1,13,9,29,49,53 \text { ) }\end{array}$ & 1 &,$- 418\left(^{* *}\right)$ &,$- 478\left(^{* *}\right)$ &, $649\left(^{* *}\right)$ &,$- 286\left(^{* *}\right)$ &,- 019 &,$- 218\left(^{* *}\right)$ &, $251\left(^{* \star}\right)$ &, $147\left(^{*}\right)$ &,$- 196\left({ }^{* *}\right)$ &, $277\left({ }^{\star *}\right)$ &,- 054 & ,032 \\
\hline $\begin{array}{l}\text { c-alumno (it. } \\
2,10,14,50,18,34 \text { ) } \\
\text { c-proces (it. }\end{array}$ &,$- 418\left(^{* *}\right)$ & 1 &, $691\left({ }^{* *}\right)$ &,$- 341\left({ }^{* *}\right)$ &, $\left.1399^{*}\right)$ & ,035 & ,037 &,$- 206\left({ }^{* *}\right)$ &,- 124 &, $186\left({ }^{* *}\right)$ &,$- 288\left({ }^{* *}\right)$ &,- 050 & ,042 \\
\hline $\begin{array}{l}7,43,11,31,15,47 \\
51)\end{array}$ &,$- 478\left(^{* *}\right)$ &, $691\left({ }^{* *}\right)$ & 1 &,$- 368\left(^{* *}\right)$ & ,126 & ,037 & ,098 &,$- 187\left({ }^{* *}\right)$ &,$- 186\left(^{* *}\right)$ &, $252\left({ }^{* *}\right)$ &,$- 219\left(^{* *}\right)$ &,- 051 &, 114 \\
\hline $\begin{array}{l}\text { c-produc (it. } \\
41,36,4,12,24) \\
\text { it } 13,62,23,75,32\end{array}$ &, $649\left(^{* *}\right)$ &,$- 341\left(^{* *}\right)$ &,$- 368\left({ }^{\star *}\right)$ & 1 &,$- 197\left(^{* *}\right)$ &,- 054 &,$- 146\left(^{*}\right)$ &, $213\left(^{* *}\right)$ &, $140\left(^{*}\right)$ &,- 134 & ,085 &,- 019 & ,018 \\
\hline $\begin{array}{l}\text { Tareas abiertas- } \\
\text { orientadas }\end{array}$ &,$- 286\left({ }^{* *}\right)$ &, $139\left(^{*}\right)$ & ,126 &,$- 197\left({ }^{\star *}\right)$ & 1 &, $170\left(^{*}\right)$ &, $362\left({ }^{\star \star}\right)$ &,$- 139\left(^{*}\right)$ &,- 005 &, $140\left(^{*}\right)$ &,- 035 &, $216\left({ }^{* *}\right)$ &, 070 \\
\hline $\begin{array}{l}\text { it } 34,63,35 \\
\text { Trabajo dirigido } \\
\text { it } 43,24,73\end{array}$ &,- 019 & ,035 & ,037 &,- 054 &, $170\left(^{*}\right)$ & 1 &, $148\left(^{*}\right)$ &,- 002 &, $214\left({ }^{* *}\right)$ &,- 005 &, 051 &, $219\left(^{* *}\right)$ &, 102 \\
\hline $\begin{array}{l}\text { Trabajo con } \\
\text { grupo-clase } \\
\text { it } 61,11\end{array}$ &,$- 218\left({ }^{* *}\right)$ & ,037 & ,098 &,$- 146\left(^{*}\right)$ &, $362\left({ }^{* *}\right)$ &, $148\left(^{*}\right)$ & 1 & ,009 &,- 119 &, $214\left({ }^{* *}\right)$ &,- 099 &, $155\left(^{*}\right)$ &, 122 \\
\hline $\begin{array}{l}\text { Enseñanza } \\
\text { expositiva } \\
\text { it } 41,33,74\end{array}$ &, $251\left(^{* *}\right)$ &,$- 206\left({ }^{* *}\right)$ &,$- 187\left(^{* *}\right)$ &, $213\left({ }^{* *}\right)$ &,$- 139\left(^{*}\right)$ &,- 002 & ,009 & 1 &, $178\left({ }^{* *}\right)$ &,- 011 &, $149\left(^{*}\right)$ &,- 030 & ,010 \\
\hline $\begin{array}{l}\text { Tareas cerradas- } \\
\text { obligatorias }\end{array}$ &, $147\left(^{*}\right)$ &,- 124 &,$- 186\left({ }^{* *}\right)$ &, $140\left(^{*}\right)$ &,- 005 &, $214\left({ }^{* *}\right)$ &,- 119 &, $178\left({ }^{* *}\right)$ & 1 &, 074 & ,108 &, $175\left(^{*}\right)$ &,- 011 \\
\hline Negociada &,$- 196\left(^{* *}\right)$ &, $186\left(^{* *}\right)$ & ,252(**) &,- 134 &, $140\left(^{*}\right)$ &,- 005 &, $214\left({ }^{* *}\right)$ &,- 011 &, 074 & 1 &,$- 472\left({ }^{* *}\right)$ & ,122 &,- 118 \\
\hline Autocratica &, $277\left(^{* \star}\right)$ &,$- 288\left(^{* *}\right)$ &,$- 219\left(^{* *}\right)$ & ,085 &,- 035 &, 051 &,- 099 &, $149\left(^{*}\right)$ &, 108 &,$- 472\left(^{* \star}\right)$ & 1 &, 000 & ,285(**) \\
\hline Explicita &,- 054 &,- 050 &,- 051 &,- 019 &, $216\left({ }^{* \star}\right)$ &, $219\left({ }^{\star *}\right)$ &, $155\left(^{*}\right)$ &,- 030 &, $175\left(^{*}\right)$ &, 122 &, 000 & &,- 071 \\
\hline Implicita &, 032 &, 042 &, 114 &, 018 &, 070 & ,102 &, 122 &, 010 &,- 011 &,- 118 &, $285\left(^{* \star}\right)$ &,- 071 & \\
\hline
\end{tabular}

** La correlación es significativa al nivel 0,01 (bilateral).

* La correlación es significante al nivel 0,05 (bilateral). 


\section{DISCUSIÓN Y CONCLUSIONES}

En primer lugar señalar, que los resultados de los análisis factoriales y la consistencia interna obtenida demuestran que las escalas utilizadas presentan un comportamiento adecuado.

En relación con el objetivo 1, se constata, en base a los resultados empíricos obtenidos mostrados en las tablas anteriores, una clara correlación entre los modelos de enseñanza/aprendizaje que se derivan de las creencias psicopedagógicas implícitas del profesor, con las tareas instructivas y de gestión del aula, de tal forma que: los modelos centrados en el profesor y el producto se relacionan significativamente con tareas instructivas de carácter expositivo y una gestión del aula autocrática. Esta relación correspondería a la perspectiva docente denominada transmisiva.

De igual manera, se aprecia también una relación positiva entre los modelos centrados en el alumno y el proceso y las tareas instructivas de carácter interactivo del tipo abiertas-orientadas y con una gestión del aula negociada. Podemos hablar así de la existencia de una perspectiva docente constructiva, definida por estas variables de pensamiento y acción de los docentes.

Por tanto, en base a los resultados obtenidos en el análisis factorial conjunto y el análisis correlacional se confirman dos perspectivas docentes bien definidas y diferenciadas: Transmisiva y Constructiva. Ahora bien, si nos fijamos en los resultados del análisis factorial conjunto (tabla 6), podemos observar como los datos apuntan que la perspectiva docente transmisiva (componente número 4) se muestra mucho más coherente ya que existe una concordancia manifiesta entre creencias y tareas instructivas. Pero, en cambio, no ocurre lo mismo con las perspectiva constructiva (componente 1) en la que no aparece ninguna tarea instructiva en relación directa con sus creencias. El resto de resultados no nos aportan información suficiente, o perfil claro, que nos permita hablar de nuevas perspectivas docentes. Como mucho, podríamos definir los otros dos núcleos de componentes incompletos (componentes 2 y 3 ). Mientras el componente 2 se comporta dentro de planteamientos de "transición" (Traver, 2000), combinando mayoritariamente tareas instructivas y de gestión de enfoques constructivos con alguna tarea instructiva transmisiva, el componente 3 desvela la soledad en la que se encuentran las tareas de gestión relacionadas con enfoques transmisivos, haciendo notar como existe una estrecha relación entre los planteamientos autocráticos y los implícitos.

En cuanto al objetivo 2, se aprecia una disonancia clara entre pensamiento y acción docente, entre creencias manifestadas y acciones instructivas explícitas. Los datos indican que existe una falta de correspondencia entre lo que los profesores "dicen que piensan" y lo que "dicen que hacen". El análisis factorial realizado sobre las creencias docentes (tabla 3) nos revela como aquellas que tienen mayor peso son las relativas a los enfoques centrados en el alumno-proceso y, en cambio, en la misma muestra de profesores el análisis factorial de tareas instructivas (tabla 4) las que mayor peso tienen son justamente las que englobamos en los enfoques opuestos de carácter transmisivo (profesor-producto). Así, se observa que los profesores presentan unas creencias predominantemente orientadas hacia el enfoque centrado en el alumno-proceso (Media $=4.42$ y Media $=4.12$ ). Sin embargo, paradójicamente, los mismos profesores manifiestan que desarrollan una enseñanza fundamentalmente expositiva y que las actividades que proponen a sus alumnos son básicamente de carácter obligatorio (Media $=2.82$ ) y que las actividades que

${ }^{4}$ Traver (2000) denomina modelos de transición docente a aquellas concepciones de la enseñanza mediante las que se reformulan los planteamientos constructivistas desde interpretaciones positivistas y que definen los caminos de tanteo que los docentes exploran al transitar entre estas dos perspectivas. 
proponen a sus alumnos son básicamente de carácter cerradas-obligatorias (Media $=2.78$ ) y de trabajo dirigido (Media= 2.43). Con respecto a las tareas de gestión el profesorado de la muestra manifiesta que la que más utilizan es de tipo explícito y negociado (tabla 5), poniendo de manifiesto que su gestión predominante en el aula es explícita (Media $=2.60$ ). Pero este tipo de tareas de gestión no va acompañada de ninguna creencia que las sustente, seguramente por ser uno de los aspectos menos trabajados en la formación. Todo ello indica que los profesores en sus acciones docentes están más cercanos al enfoque profesor-producto, contrariamente a lo manifestado en sus creencias. A la luz de estos resultados la pregunta que surge es la siguiente ¿por qué los profesores no desarrollan en el aula unas acciones docentes en concordancia con sus teorías y creencias pedagógicas? Posiblemente, la perspectiva Constructiva que se refleja en las creencias de la mayoría del profesorado de la muestra, forma parte del discurso psicopedagógico difundido a partir de la promulgación y puesta en marcha en España de la LOGSE y, por tanto, es un discurso que ha ido calando progresivamente, desde 1990, en el pensamiento del profesorado, a través de la formación inicial y permanente desarrollada a lo largo de todos estos años. Sin embargo, la perspectiva Transmisiva sigue siendo en la actualidad la más habitual y claramente definida en la realidad educativa reflejada en la acción docente, bien porque el profesorado no posee la formación y habilidades docentes necesarias (autoeficacia) para llevar a la práctica sus teorías y creencias (Marchesi, 2000), bien porque existen obstáculos que interfieren la puesta en práctica de sus teorías y creencias, bien porque no cuenta con los apoyos y recursos materiales y personales necesarios en el propio centro, etc. Estas son cuestiones que ya hemos abordado en investigaciones recientes (Doménech, Traver, Moliner y Sales, 2005) y que se seguirán desarrollando en el futuro.

De estas conclusiones podemos inferir algunas implicaciones pedagógicas que sería interesante seguir investigando y desarrollar en futuros trabajos derivados de esta investigación:

- La comprobación de que se da una correlación directa y positiva entre las tareas instructivas y de gestión del aula que definen la acción docente, nos alerta de la necesidad de darle una nueva concepción y sentido al espacio del aula. El aula como lugar de intercambio de experiencias y de cultura, de implicación, autoconomiento, autonomía, comunicación y socialización, abierto al conjunto de diversidades que presenta el alumnado (Masip y Rigol, 2000). Debe ser un espacio de participación activa, de interacción y diálogo en el que la aceptación, el cuestionamiento, el rechazo y la asunción de procesos va a ser lo que orientará el proceso de enseñanza-aprendizaje y determinará también el clima cultural y social de la misma (Arnáiz e Illán, 1996). Ya no podemos considerar el aula como un espacio cerrado y autosuficiente, sino en un lugar de puesta en común y diálogo en el que se va construyendo el conocimiento a partir de las experiencias sobre la realidad y de la mediación de los otros, adultos e iguales.

- Situados en este contexto, se abren nuevas cuestiones en las que enmarcar el conocimiento y la práctica docente desde un enfoque ecológico de las perspectivas docentes. No se pueden obviar los contextos institucionales ni las dimensiones de carácter más organizativo, social e ideológico que también conforman la construcción y el ejercicio de la profesión docente (Bolívar, 1995).

- Estamos hablando, pues, de la necesidad de definir un nuevo perfil docente, de un profesorado reflexivo y crítico que parte de su práctica para confirmar o modificar sus teorías, sin por ello 
despreciar los conocimientos que la teoría y la técnica le pueden aportar. Su reflexión en la acción exige una actitud investigadora para así ir ajustando sus previsiones y modificar sus teorías si fuera necesario. Tal como define Zeichner (1993) se trata de un profesional abierto al diálogo, a la deliberación grupal, a la escucha de opciones distintas a la suya. Por ello, la comunicación, la colaboración y la deliberación entre profesionales de la educación, en equipos docentes, son procedimientos, junto al análisis constructivo de la propia actividad docente, para el cambio y la transformación de las prácticas educativas.

Así pues, la interrelación entre tareas instructivas y la gestión del aula, la necesidad de un enfoque ecológico del pensamiento y acción docente y la conveniencia de definir un nuevo perfil profesional del profesorado nos plantea la superación de la perspectiva docente transmisiva, predominante todavía en las prácticas educativas y comenzar procesos formativos y de desarrollo profesional del profesorado que le permitan acercarse hacia perspectivas más constructivistas, ambiguas aún en las actuaciones desarrolladas por los profesores de Secundaria, tal y como muestra el presente trabajo. Desde hace ya algún tiempo los planteamientos de las perspectivas sociocomunitarias en educación trabajan en esta línea. Desde este punto de vista se hace necesario aunar teoría y práctica pedagógica, anclándolas en la realidad-aula donde se desarrolla la acción docente.

En este sentido, debemos seguir estudiando este proceso de cambio y sus implicaciones en la formación inicial y permanente del profesorado, en futuras investigaciones derivadas de la que hemos desarrollado en el presente artículo.

\section{REFERENCIAS BIBLIOGRÁFICAS}

Angulo, F. y PéRez Gómez, A.I. (coords.) Desarrollo profesional del docente: Política, Investigación y Práctica. Madrid: Akal, 1999.

Arnálz SÁnchez, P. e ILLÁN Romeu, N. Procesos de enseñanza-aprendizaje ante las necesidades educativas especiales, en ILLÁN ROMEU, N. (Coord.): Didáctica y organización en la Educación Especial. Málaga: Aljibe, 1996.

BolíVAR, A. El conocimiento de la enseñanza. Epistemología de la investigación curricular. Granada, Universidad de Granada/ FORCE, 1995.

Clark, C. M. y Peterson, P. L. Procesos de pensamiento de los docente, en WiTTROCK, M. C. La investigación de la enseñanza, III. Profesores y alumnos. Barcelona: Paidós Educador, 1989.

COLL, C. y MARTí, E. Aprendizaje y desarrollo: la concepción genético-cognitiva del aprendizaje, en COLL, C.; PALACIOS, J. y MARCHESI, A. (Comps.) Desarrollo psicológico y educación, Il. Psicología de la educación. Madrid: Alianza editorial, 1991.

DíAz-Aguado, M. J. Estilos de enseñanza, en BELTRÁN, J. Psicología Educacional. Madrid: UNED, 1985.

DOMÉNECH, F. El proceso de enseñanza/aprendizaje universitario. Aspectos prácticos y teóricos. Castelló: Publicacions de la Universitat Jaume I, 1999c.

DOMÉNECH, F. Las teorías educativas implícitas del profesorado universitario. III Congreso Internacional de Psicología y Educación. Santiago de Compostela, 8-11 de septiembre, 1999a. 
DOMÉNECH, F. Nivel de concordancia entre las teorías educativas del profesor universitario y su conducta de clase. III Congreso Internacional de Psicología y Educación. Santiago de Compostela, 8-11 de septiembre, $1999 b$.

DOMÉNECH, F. Psicología de la educación e instrucción: su aplicación al contexto de la clase. Castelló: Universitat Jaume I, 2004.

DOMÉNECH, F. y GÓmEZ, A. Las creencias psicopedagógicas de los futuros profesores de Secundaria y su relación con las demandas de examen y con la organización espacial de la clase, Revista de Investigación Educativa, 21, (2), 489-505. (2003).

Doménech, F.; Traver, J. A.; Moliner, O. y Sales, A. Análisis de las variables mediadoras entre las concepciones educativas del profesor de secundaria y su conducta docente, en Revista de Educación (en prensa), 2005.

DOMĖNECH, J. y ViÑAS, J. La organización del espacio y del tiempo en el centro educativo. Barcelona: Graó, 1997.

FERRÉs, J. Educar en una cultura del espectáculo. Barcelona: Paidós, 2000.

FUÉGUEL, Cora Interacción en el aula. Estudio de caso. Barcelona: Praxis, 2000.

KEMBER, D. A reconceptualistion of the research into university academics' conceptions of teaching, Learning and Instruction, 7, 255-275, 1997.

MARCHESI, A. Controversias de la educación españolas. Madrid: Alianza Editorial, 2000.

Martín Bris, M.; MARgalef, L. y Rayón, L. La respuesta a la diversidad en la Enseñanza Obligatoria: los modelos de planificación y organización. Alcalá de Henares: Servicio de Publicaciones de la Universidad de Alcalá de Henares, 2000.

MASIP, M. y RIGOL, A. El aula, escenario de la diversidad, en VARIOS ¿Cómo hacerlo? Propuestas para educar en la diversidad. Barcelona: Graó, 2000.

Montero, M. L. Los estilos de enseñanza y las dimensiones de la acción didáctica, en COLL, C.; PALACIOS, J. y MARCHESI, A. Desarrollo psicológico y educación, II. Psicología de la educación. Madrid: Alianza Editorial, 1991.

NORUSIS, M.J. SPSS for Windows (Versión 10.0) Chicago; IL: SPSS Inc., 1999.

PéREZ PÉREZ, C.) Las normas en el curriculum escolar. Madrid: Eos, 1996.

RUÉ, J. El aula. Un espacio para la cooperación, en MIR, C. (Coord.) Cooperar en la escuela. La responsabilidad de educar para la democracia. Barcelona: Graó, 1998.

Sales Ciges, A. Atención a la Diversidad. Construyendo la escuela inclusiva e intercultural. Proyecto Docente, Valencia, 2004.

TABACHNICK, B. y ZEICHNER, K. The impact of the student teaching experience on the development of teacher perspectives, en TABACHNICK, B. y ZEICHNER, K. The Development of Teacher Perspectives: Final Report. Wisconsin: Wisconsin Center for Education Research, 1985. 
Traver Martí, J. A. Diferencias individuales y aprendizaje escolar. La diversidad como problema, en ADRIÁN, J. E. y CLEMENTE, Rosa A. (Ed.) Convivencia escolar en secundaria. València: Generalitat Valenciana. Conselleria de Cultura, Educació i Esport, 2004.

Traver Martí, J. A. Trabajo cooperativo y aprendizaje solidario. Aplicación de la Técnica Puzzle de Aronson para la enseñanza y el aprendizaje de la actitud de solidaridad. Tesis Doctoral. Departament d’Educació. Facultat de Ciències Humanes i Socials. Universitat Jaume I, Castelló (en prensa), 2000.

URíA, E. Estrategias didáctico-organizativas para mejorar los centros educativos. Madrid: Narcea, 1998.

VARIOS Una organización sometida a vaivenes, Cuadernos de Pedagogía, 326, julio, 95-98, 2003.

ZEICHNER, K.M. (1993) El maestro como profesional reflexivo, Cuadernos de Pedagogía, 220, 44-49.

itraver@edu.uji.es; asales@edu.uji.es; betoret@psi.uji.es; molgar@edu.uji.es 


\title{
Contactar
}

Revista lberoamericana de Educación

\author{
Principal OEI
}

